\section{$\underset{\substack{\text { hommes } \\ \text { \& migrations }}}{ }$}

\section{Hommes \& migrations}

Revue française de référence sur les dynamiques

migratoires

$1305 \mid 2014$

L'exil chilien en France

\title{
Le Chili et les mouvements migratoires
}

\section{Ricardo Parvex}

\section{(2) OpenEdition \\ Journals}

\section{Édition électronique}

URL : http://journals.openedition.org/hommesmigrations/2720

DOI : 10.4000/hommesmigrations. 2720

ISSN : 2262-3353

\section{Éditeur}

Musée national de l'histoire de l'immigration

\section{Édition imprimée}

Date de publication : 1 janvier 2014

Pagination : 71-76

ISBN : 978-2919-040261

ISSN : $1142-852 X$

\section{Référence électronique}

Ricardo Parvex, "Le Chili et les mouvements migratoires », Hommes \& migrations [En ligne], 1305 | 2014, mis en ligne le 01 janvier 2017, consulté le 19 avril 2019. URL : http://

journals.openedition.org/hommesmigrations/2720; DOI : 10.4000/hommesmigrations.2720 


\title{
LE CHILI ET LES MOUVEMENTS MIGRATOIRES
}

par RICARDO PARVEX, vice-président de l'Association des ex-prisonniers politiques chiliens-France.

\author{
Depuis le XVe siècle, I'histoire du Chili s'est construite autour \\ des migrations de peuplement. Si elle était au départ constituée \\ par des mouvements de populations internes au continent \\ américain, l'immigration des Européens au Chili commence avec \\ la conquête espagnole. Elle se poursuit notamment aux XIXe et \\ XXe siècles par l'arrivée de populations venues de toute l'Europe \\ cherchant au Chili une terre d'accueil. La dictature de Pinochet \\ va susciter d'importants flux en sens inverse : une partie \\ de la population quittera le pays, contribuant à ouvrir le Chili \\ au reste du monde.
}

Comme tous les pays du continent américain, le Chili, avant la colonisation, était habité par une population autochtone dont les origines sont source d'interminables discussions. Toutefois, il existe aujourd'hui un consensus autour de l'idée que le gros des premiers habitants américains est arrivé d'Asie par le détroit de Behring il y a environ quinze mille ans.

La préhistoire du continent montre que des migrations internes existent depuis longtemps. Celle qui touche de plus près la population originaire du Chili est le déplacement et la colonisation forcés du territoire du Tihuantinsuyo ${ }^{1}$ opérés par l'Empire inca pendant le $X V^{\text {e }}$ siècle. Afin d'organiser l'ad- ministration et l'exploitation de ses vastes possessions, la monarchie impériale de Cusco arrachait certaines populations à leurs régions d'origine en les installant sur des territoires qu'elle voulait coloniser et contrôler. Ces colonisateurs forcés étaient appelés "mitimaës" et le Chili en a reçu un certain nombre.

Avant la conquête espagnole, les territoires des actuels Équateur, Pérou et Bolivie, ainsi que le Sud colombien et le Nord argentin et chilien appartenaient à l'Empire inca. L'arrivée des Européens est venue se superposer aux migrations commandées juste quelques décennies plus tôt par le gouvernement impérial. 


\section{La conquête et la colonisation européennes}

Les historiens ont toujours été surpris par le faible nombre d'hommes et la modestie des moyens qui ont suffi aux conquistadors espagnols pour dominer de vastes populations indigènes, y compris de grands empires tels celui des Incas ou des Aztèques. Effectivement, la première conquête militaire espagnole, qui n'avait pas d'objectif de peuplement mais seulement de spoliation de richesses existantes et facilement transportables en Europe (or, argent et pierres précieuses, bijoux et parements liturgiques et cérémoniaux, etc.), n'était constituée que d'une poignée d'hommes ambitieux et résolus.

Les choses ont changé lorsque les conquistadors ont compris que l'exploitation de beaucoup de ces richesses demandait la prospection et la recherche de filons miniers, l'exploita-

On estime qu'environ tion de mines, l'orpaillage, 800000 Chiliens seraient d'ascendance française. La plupart de ces immigrants ont migré de manière individuelle ou familiale. le transport de minerais, etc., activités consommatrices d'une main-d'œuvre nombreuse, nécessitant un encadrement important. Très rapidement, la simple conquête de spoliation à faible contingent est devenue de la colonisation massive avec implantation durable et peuplement européen permanent. Quelques années après l’arrivée des Espagnols en Amérique latine et au Chili, ceux-ci constituaient la première population immigrée du territoire.

Dans les pays tropicaux producteurs de denrées agricoles de rente telles que la canne à sucre, le coton, le café, le tabac, etc., les besoins en force de travail ont dépassé les possibilités offertes par une population indienne en voie d'extinction à cause des mauvais traitements, des épidémies de peste et autres maladies contagieuses. Les arrivages massifs d'esclaves africains viennent apporter la main-d'œuvre nécessaire à ce commerce d'exporta- tion en pleine croissance. L'immigration forcée est à l'origine de l'importante population noire présente actuellement en Amérique latine. Le Chili, étant un pays tempéré sans cultures tropicales, n'a reçu des Africains que très marginalement. Ils ne constituent à l'heure actuelle qu'une population noire indécelable.

\section{Le Chili, pays d'accueil de migrants}

Comme tous les pays du Cône sud de l'Amérique latine, le Chili a accueilli depuis des migrants venus de divers horizons, notamment des Européens du Sud. C'est une évidence que la plus importante population immigrante dans le pays a été espagnole. En ne prenant en considération que les immigrants d'origine européenne, nous devons ajouter les Italiens, 600000 Chiliens étant de cette origine selon les estimations actuelles ; les Grecs, avec plus de 100000 descendants directs, en particulier à l'extrême nord (Antofagasta) et à l'extrême austral (Punta Arenas). Comparativement, cela fait du Chili l'un des cinq pays ayant le plus grand nombre de descendants de Grecs au monde. On estime, par ailleurs, à environ 400-500 000 le nombre de Chiliens descendant de Croates, ce qui fait du Chili l'un des plus importants centre d'accueil de Croates du monde.

L'importance de l'immigration française n'apparaît pas liée à une activité, à une époque ou à une région particulière, c'est pourquoi elle est moins visible. Cependant, le Chili est peut-être le pays latinoaméricain où l'influence française se fait sentir de la manière la plus claire. On estime qu'environ 800000 Chiliens seraient d'ascendance française.

La plupart de ces immigrants ont migré de manière individuelle ou familiale. Dans la grande majorité des cas, ils ont utilisé des filières de parenté ou des liens géographiques et professionnels. Il existe cependant plusieurs exemples d'arrivées massives d'Européens organisées officiellement 
par les autorités chiliennes. C'est le cas des colons allemands recrutés en Allemagne et amenés au Chili par Vicente Pérez Rosales, agent du gouvernement de Santiago vers la moitié du XIX siècle. La loi d'“immigration sélective", édictée par le gouvernement en 1845, a permis à ces immigrants de coloniser et de peupler de vastes régions du Sud chilien (Valdivia, Osorno, Llanquihue). À l'heure actuelle, les autorités estiment que le nombre de descendants de ces premiers colons oscille entre 500 et 600000 .

Les autres cas d'arrivées organisées ont été l'accueil des réfugiés républicains après la fin de la guerre d'Espagne (1939) et celui des juifs d'Europe centrale et de l'Est (Tchèques, Polonais, Hongrois) à la veille de la Seconde Guerre mondiale.

Le cas des Espagnols correspond à une initiative du gouvernement dirigé par la coalition du Front populaire chilien pour venir en aide à quelque 2500 réfugiés républicains chassés de la péninsule par la dictature franquiste. À ces réfugiés arrivés dans le bateau Winnipeg, se sont ajoutés des milliers d'autres Espagnols ayant des liens familiaux, politiques ou professionnels avec les premiers.

En ce qui concerne les "juifs de l'Est", l'accueil est resté bien plus modeste, comparé à celui mis en place en Argentine depuis la fin du XIX siècle.

Dans le cas des non-Européens, l'immigration palestinienne et syro-libanaise est en tête avec plus d'un demi-million de descendants directs. Le Chili possède la première communauté palestinienne du monde hors Palestine.

\section{Les migrations chiliennes au sein du Cône sud}

La place du Chili dans la géographie sud-américaine et mondiale a fait de cette nation une espèce d'“île en terre ferme". Séparés de leur proche voisin argentin par la deuxième chaîne de montagnes la plus haute du monde, face à l'océan Pacifique qui met les côtes les plus proches (Chine, Austra- lie, etc.) à des milliers de kilomètres, avec un grand désert au nord, et au sud le cap Horn et l'Antarctique, les Chiliens ont été traditionnellement plutôt une population "sédentaire et installée". En dehors de quelques centaines de Chiliens ayant fait le voyage en Californie à l'époque de la fièvre de l'or, jusqu'à la fin du $\mathrm{XX}^{\mathrm{e}}$ siècle, l'histoire n'enregistre pas d'autres migrations chiliennes massives. Cela sans tenir compte des milliers de Chiliens qui se sont installés au sud et en Patagonie La place du Chili dans la géographie sud-américaine et mondiale a fait de cette nation une espèce d'"île en terre ferme". argentine depuis des décennies.

En effet, les populations du sud du Chili et celles de la Pampa et de la Patagonie argentines partagent une culture et un peuple communs, le peuple Mapuche. Depuis des siècles, cette ethnie a pratiqué une vie nomade à travers la cordillère des Andes qui sépare ces deux territoires. Pendant tout le XIX ${ }^{e}$ siècle, on trouve leurs tribus aussi bien en Argentine qu'au Chili. Après l'écrasement violent et la soumission des Mapuche menés simultanément par les deux gouvernements dans la deuxième moitié du XIX siècle, les migrations à travers les Andes ont changé de nature, ont diminué, mais n'ont pas cessé. Le territoire argentin, plus vaste et plus riche que celui du Chili a attiré davantage les populations de paysans pauvres, descendants directs de Mapuche et de métis du Sud chilien.

\section{Les migrations de l'exil}

Cette situation a brusquement changé dans les années 1970. Tout d'abord, l'élection d'un gouvernement de gauche en 1970 créa un certain émoi parmi les classes possédantes dont une infime partie quittèrent le pays pour l'Argentine, le Brésil ou les États-Unis afin de protéger leur argent. La chute de ce gouvernement suite au coup d'État militaire de septembre 1973 a complètement changé la situation. La répression brutale, les exécutions sommaires, les persécutions massives et 
les milliers d'arrestations d'opposants ont systématiquement semé la terreur et chassé une partie significative de la population, notamment les jeunes générations engagées dans le processus de changement.

Pour la première fois, le pays a vu partir des milliers de personnes vers les destinations les plus diverses. Il s'agissait tout d'abord de "se mettre à l'abri". Le choix de la destination n'était pas le principal critère retenu. Les choses se sont passées plutôt de manière inverse. Horrifiés par la violence de la dictature militaire chilienne,

Les principaux pays d'accueil

en Europe, par ordre

d'importance, ont été la Suède, la France, I'Italie, l'Allemagne,

la Grande-Bretagne,

le Danemark, la Belgique, les Pays-Bas, etc. de nombreux pays ont ouvert leurs frontières pour recevoir des Chiliens qui sortaient de prison ou qui étaient poursuivis. Les principaux pays d'accueil en Europe, par ordre d'importance, ont été la Suède,

la France, l'Italie, l'Allemagne, la Grande-Bretagne, le Danemark, la Belgique, les Pays-Bas, etc. Toutes ces nations, sans compter les "démocraties populaires" du centre et de l'est de l'Europe - qui ont mis en place un système d'accueil et d'exil pour leurs alliés politiques chiliens -, ont reçu des milliers de Chiliens à partir de 1973 et pendant les dix-sept ans de la dictature. Même l'Espagne, encore sous le régime franquiste, a dû se résoudre à recevoir de nombreux exilés chiliens disposant de la double nationalité, dont bénéficient tous les descendants directs de citoyens espagnols.

\section{Un accueil facilité en France}

L'accueil que les exilés chiliens ont reçu en Europe serait inconcevable aujourd'hui. Les facteurs qui ont contribué à le rendre humain et solidaire ont changé ou simplement n'existent plus.

Le premier de ces facteurs a été l'état de l'économie française et européenne. Entre 1973 et 1976, années d'arrivée d'importants contingents de réfu- giés, le Vieux Continent bénéficiait encore des beaux restes de cette période faste que furent les Trente Glorieuses : un bon taux de croissance, le plein emploi, un faible endettement, un excédent commercial, et leur corollaire : des garanties assurées pour les salariés et leurs familles, la présence redistributive et protectrice de l'État, les programmes sociaux, etc. L'aisance que la prospérité des Trente Glorieuses apportait à la France a donc été un facteur très important pour cet accueil. À l'époque, la France était une nation qui croyait encore majoritairement au partage, surtout avec les pourchassés que nous étions. Aujourd'hui, la France est bien plus riche qu'il y a quarante ans, mais le sens de la solidarité collective et du partage semble avoir péri entre les mains de l'individualisme et de l'efficacité économique. Le deuxième facteur contribuant à faire de cet accueil une véritable chance d'insertion pour les exilés chiliens et latino-américains a été la réalité politique et le rapport de forces entre les différents courants présents sur l'échiquier politique français. À l'époque, l'Union de la gauche était donnée gagnante aux échéances électorales de la période. Le Parti communiste français, au sommet de sa puissance, montrait une solidarité sans limites vis-à-vis des "frères chiliens victimes du fascisme et de l'impérialisme". Les Chiliens ont bénéficié d'un accueil extraordinaire. Leur ont été proposés des cours de formation et de langue, des bourses d'étude, l'accès à des logements HLM et même à des postes de travail quand cela était possible. Mais le plus important, c'était l'écoute. Nous avons été écoutés, suivis, accompagnés...

\section{La noblesse de la cause des exilés chiliens}

Parmi les conditions du bon accueil des Chiliens, il faut citer le prestige et la noblesse que l'opinion publique française et internationale voyait dans la lutte de notre peuple. Notre combat pour la justice sociale, le triste sort auquel les puissants nous 
avaient condamnés, ajouté au sacrifice héroïque de nos dirigeants, tout cela ne pouvait que susciter et renforcer la solidarité à notre égard.

Il ne faut pas oublier qu'après le mouvement de mai 1968, beaucoup de jeunes Français de cette génération étaient en quête de "modèles". Pour continuer à croire "aux lendemains qui chantent", ils avaient besoin de pistes politiques crédibles. Le Chili d'Allende, avec sa vaste coalition démocratique et pluri-classiste exprimée par l'Unité populaire, représentait aux yeux de beaucoup de Français et d'Européens ce modèle tant souhaité. Des politologues sérieux pensaient qu'une coalition politique comme celle de l'Unité populaire chilienne aurait pu constituer un exemple de poids pour créer et construire le "Programme commun” de la gauche française. Pendant le gouvernement d'Allende, la diversité politique a été la principale caractéristique de la vivacité démocratique de la période. De la social-démocratie du centre jusqu'aux mouvements révolutionnaires de l'extrême gauche en passant par les radicaux, les socialistes, les communistes et les chrétiens progressistes, tous agissaient et s'exprimaient librement. Une fois en exil, toutes ces sensibilités ont trouvé leurs pairs et leurs homologues européens. Cela a permis que chacun des secteurs politiques français ait ses propres "exilés chiliens" à soutenir.

La nature sociologique des exilés chiliens arrivés en France (officiellement moins de 10 000), majoritairement des intellectuels, des jeunes professionnels, des cadres politiques, etc., a contribué à faire d'eux, malgré leur nombre réduit, les animateurs d'une certaine vie sociale et culturelle à la fin du XXe siècle.

Quarante ans plus tard, nous constatons que les anciens exilés vivant encore dans l'Hexagone (aujourd'hui Français ou résidents permanents pour la plupart) ou leurs enfants ont conservé pour l'essentiel leur sensibilité progressiste, même si elle ne s'exprime pas toujours de façon publique ou militante. Malgré les difficultés de réintégration, pour ceux qui sont rentrés au pays (entre un tiers et $40 \%$ des exilés), ils ont fini par "redevenir" des Chiliens. Ces familles constituent actuellement un pont ouvert sur la culture internationale ce qui a permis au Chili de rompre l'isolement auquel la nature et la géographie semblaient l'avoir condamné.

\section{La place de l'exil dans I'histoire contemporaine du Chili}

De la même façon que les tortures, les disparitions et les exécutions de prisonniers, l'exil a été un sujet tabou pendant tout le premier temps dela dictature, malgré cette réalité inédite pour un pays "sédentarisé" commele Chili : lémigration massive après le 11septembre1973. Chaquefamillechilienneaperdu une partie de ses membres du fait de la répression, de l'exil ou des deux. L'Église catholique a beaucoup communiqué sur ce thème et aidé à ce que l'opinion publique chilienne prenne conscience de la gravité de cette punition.

Plusieurs polémiques ont De la même façon que émergésurcetexildepresque les tortures, les disparitions et un million de Chiliens (entre les exécutions de prisonniers, 800000 et 1000 000).

L'un des points controversés semble avoir été relevé par les milieux proches du pouvoir dictatorial. Il consiste à présenter l'exil comme une espèce de cadeau fait aux l'exil a été un sujet tabou pendant tout le premier temps de la dictature, malgré cette réalité inédite pour un pays "sédentarisé" comme le Chili : l'émigration massive après le 11 septembre 1973. forgé une expression, "la bourse Pinochet", pour signifier que les milliers de Chiliens expulsés directement ou indirectement du pays ont été en réalité les "bénéficiaires" d'une sorte d'aide sponsorisée par la tyrannie. Pour une population peu habituée à s'expatrier et à parcourir le monde, le fait de quitter le pays et d'être accueilli à l'étranger a été présenté comme un "privilège". Malheureusement, ce mensonge a si bien marché que même des milieux proches de l'exil le répètent parfois. 
C'est oublier que le bannissement est une peine de longue durée, une punition qu'on transmet aux enfants et aux familles qui n'ont aucune raison de subir, par extension, ce châtiment. C'est aussi ignorer que si l'exil existe depuis la nuit des temps et que s'il perdure jusquà nos jours, c'est bien parce qu'il s'agit d'une punition efficace qui a fait ses preuves depuis Babylone et la Grèce antique.

Le deuxième point de controverse sur l'exil s'est ouvert très tôt parmi les exilés eux-mêmes. Il s'agissait de distinguer les "véritables" exilés politiques de ceux qui n'auraient été que des exilés économiques. Dans la première catégorie, on incluait tous ceux qui avaient été emprisonnés, torturés ou qui, étant recherchés, ont pu s'enfuir avant d'être capturés. Dans la catégorie économique, on classait ceux qui, n'ayant été ni emprisonnés, ni poursuivis, ni apparemment menacés, adoptaient le statut de réfugié.

Si l'on regarde de plus près, nous nous rendons compte qu'il s'agit là d'une fausse polémique. Si nous passons en revue les statistiques démographiques du Chili, nous constatons très vite que jusqu'en 1970 le pourcentage de la population chilienne qui quittait le pays a été infinitésimal. Après l'élection de l'Unité populaire avec Salvador Allende au gouvernement, un groupe extrêmement réduit de grandes fortunes, prévoyantes ou effrayées, a quitté temporairement le pays. Mais cela était statistiquement presque indétectable.

En revanche, c'est à partir du 11 septembre 1973 que commence une véritable vague de départs, tendance qui ne fera qu'augmenter au fur et à mesure que la dictature accroît sa brutalité et sa violence. Cependant, cette violence ne se manifestait pas seulement par les arrestations, les tortures et les violations massives des droits de l'homme. Elle se mettait en place surtout d'un point de vue économique et social : fermeture en chaîne d'usines et de centres de travail, licenciements massifs, coupures budgétaires des programmes d'aide sociale, réorganisation néo-libérale de la production et de la propriété, etc. Ces mesures ont créé un chômage inédit dans le pays, aggravé par le fait que toute contestation, même pacifique, était interdite et punie par la prison ou la mort. Dans ces conditions, il n'est pas étonnant qu'une partie importante de la population, notamment les jeunes générations, ait été obligée de quitter le pays, s'ajoutant à la masse des réfugiés de type politique présente un peu partout dans le monde. Il s'agit par conséquent bel et bien d'un exil dont la racine est nettement politique, même si c'est par le biais indirect de la répression économique et sociale. Dans divers pays tels que le Canada, l'Australie, la Nouvelle-Zélande, ces réfugiés économiques ont reçu le statut de migrant et pas d'exilé.

\section{Épilogue}

Une fois la dictature finie, une longue et interminable transition vers la démocratie a commencé en 1990. À partir de ce moment-là, le flux de Chiliens quittant le territoire national a cessé presque complètement.

Il reste cependant un héritage de cette période : les liens familiaux, professionnels et sociaux culturels tissés entre les Chiliens de l'exil et leurs terres d'accueil. La mondialisation aidant, leur mobilité est aussi assurée par les contacts économiques, culturels et commerciaux qui les font se déplacer vers divers points de la planète. C'est ainsi que de jeunes professionnels, des étudiants, des stagiaires, des artistes voyagent et s'installent transitoirement ou de manière durable un peu partout dans le monde.

Néanmoins, ces déplacements et ces expatriations ont peu à voir avec le caractère punitif qu'a représenté l'exil sous la dictature militaire. De la même manière, le Chili est une terre d'accueil et d'enracinement pour un nombre toujours croissant d'étrangers. Ainsi, les déchirements et l'évolution historique du pays pendant la deuxième moitié du $\mathrm{XX}$ siècle ont contribué à désenclaver cette "île en terre ferme" qu'est le Chili. 